\title{
Optimisation of Arsenic (III) by Colorimetric Incorporated with Image Processing Technique
}

\author{
JIN HOONG LEONG ${ }^{1}$, KEAT KHIM ONG ${ }^{2 *}$, WAN YUNUS WAN MD ZIN³, \\ FITRIANTO ANWAR ${ }^{4}$, UMMUL FAHRI ABDUL RAUF ${ }^{2}$, CHIN CHUANG TEOH ${ }^{5}$, \\ HUSSIN ABDUL GHAPOR ${ }^{3}$ and MOHD JUNAEDY OSMAN ${ }^{2}$ \\ ${ }^{1}$ Faculty of Engineering, Universiti Pertahanan Nasional Malaysia, \\ Kem Sungai Besi, 57000 Kuala Lumpur, Malaysia. \\ ${ }^{2,3}$ Centre for Defence Foundation Studies, Universiti Pertahanan Nasional Malaysia, \\ Kem Sungai Besi, 57000 Kuala Lumpur, Malaysia. \\ ${ }^{4}$ Faculty of Science, Universiti Putra Malaysia, 43400 UPM Serdang, Selangor, Malaysia. \\ ${ }^{5}$ Malaysian Agricultural Research and Development Institute Headquarter, \\ G. P.O. Box 12301, 50774 Kuala Lumpur, Malaysia. \\ *Corresponding author E-mail: ongkhim @ upnm.edu.my \\ http://dx.doi.org/10.13005/ojc/320512
}

(Received: September 05, 2016; Accepted: October 12, 2016)

\begin{abstract}
Inorganic arsenic contamination has caused a remarkable impact on the contamination of soil and groundwater in many countries. Consequently, determination of inorganic arsenic on site is very crucial especially arsenic (III) which is more toxic than arsenic (V). Thus, a more rapid, simple and ecofriendly approach was developed in this study to determine arsenic (III) by incorporation of image processing technique into colorimetric method. The effects of various factors were evaluated by a $2^{4}$ full factorial design with a blocking factor. The mass ratio of sulfamic acid to zinc powder was the most significant factor affected red, green and blue (RGB) color values and followed by reaction period. The optimum conditions for the detection were found to be using $1 \mathrm{~g}$ of sulfamic acid and $0.5 \mathrm{~g}$ of zinc powder at 5 minutes. This work also demonstrates that the developed method is able to detect arsenic (III) rapidly and easily.
\end{abstract}

Keywords: Arsenic (III), Colorimetric, Factorial design analysis, Image Processing, Optimisation.

\section{INTRODUCTION}

Continuous exposures to toxic chemicals for example arsenic could cause a significant risk to public health ${ }^{1}$ as it is a highly toxic element even at low concentration ${ }^{2}$. Arsenic occurs in nature water in various forms of inorganic and organic ${ }^{3}$ and mainly found in two inorganic forms i.e. $\mathrm{As}^{3+}$ and $\mathrm{As}^{5+}$, whereby As (III) is more toxic than As (V) $)^{4}$. According to Shen et. al $(2013)^{5}$, As (III) is able to bind to a specific protein which could alter the protein's conformation, leading to a deterioration in cellular functions. In addition, based on the report by Bednar et al. (2004) $)^{6}$ determination of each inorganic arsenic 
species is crucial due to the extensive variation in the toxicology, mobility, and absorptivity of each species.

Laboratory instruments can provide highly accurate and precise analysis to measure arsenic in water samples, but it is time consuming and costly, consequently various arsenic test kits have been developed based on colorimetric method. However the results obtained is normally based on comparison of the color formed on the test strip with a reference color scale which is rather subjective and operatordependent. Thus there is a need for improvement for in-situ analysis of arsenic. According to Wang et al. $(2011)^{7}$, an ideal field deployable sensor would be able to detect low $\mu \mathrm{g} / \mathrm{L}$ concentration of analyte directly on-site with little or no sample preparation as well as persistence to interference ions. In fact, colorimetric methods can provide results nearly as accurate and reliable as those from analytical laboratories when the reaction is automatically evaluated by means of a color detector ${ }^{8}$. An electronic device for measuring color has been introduced to minimize human error in interpreting the color with naked eyes for determination of arsenic. For example, Anderson et al. (2008) ${ }^{9}$ measured reflectance of the developed color spot and convert it to digital signal by an electronic transducer which requires 30 to 40 minutes to detect of arsenic.

Thus, a more rapid, simple and ecofriendly approach was developed in this study to determine arsenic (III) by incorporation of image processing technique into colorimetric method. The effects of different factors were investigated by a $2^{4}$ full factorial design with a blocking factor. The effects consists of weight load used for drying silver nitrate-impregnated filter paper, drying period of silver nitrate-impregnated filter paper, mass ratio of sulfamic acid to zinc powder and reaction period between arsine gas generated and silver nitrate which were evaluated at two levels to determine the significant factors before optimize the detection of arsenic (III).

\section{MATERIALS AND METHODS}

\section{Reagents and Materials}

Arsenic (III) stock solution containing 1,000 mg As (III)/L (Merck, Germany) was used to prepare As (III) working standard solutions. Sulfamic acid, silver nitrate and zinc powder were also obtained from Merck (Germany). All the chemicals used in this study were of analytical grade.

\section{Preparation of As (III) working standard solutions \\ As (III) working standard solutions containing} 0 to $300 \mu \mathrm{g} / \mathrm{L}$ of As (III) were freshly prepared from the As (III) stock solution by proper dilutions using ultrapure water.

\section{Preparation of $5 \%(\mathrm{w} / \mathrm{v})$ silver nitrate solution}

A $5 \%$ solution of silver nitrate was prepared by dissolving the silver nitrate using ultrapure water in a $100 \mathrm{~mL}$ volumetric flask.

\section{Preparation of silver nitrate-impregnated filter paper}

Whatman filter paper No.3 was cut into a $2.5 \mathrm{~cm}$ (diameter) round-shaped piece of the paper. It was then dipped into the silver nitrate solution for period of 2 seconds and followed by drying it between two pieces of dry Whatman filter papers which was pressed using a 100 or $500 \mathrm{~g}$ load for 20 or 60 seconds. This silver nitrate-impregnated filter paper was used as arsine sensor paper.

\section{Colored complex formation}

Minitab software (version 17.0) (USA) was utilized to randomize the ninety-six experimental runs with all possible combinations of factors in duplicates at high and low levels to investigate the effect of weight load (100 or $500 \mathrm{~g}$ ) used for drying silver nitrate-impregnated filter paper (DW), drying period of silver nitrate-impregnated filter paper (DP) (20 or $60 \mathrm{~s}$ ), mass ratio of sulfamic acid to zinc powder (MSZ) (1.0 g: $0.5 \mathrm{~g}$ or $4.0 \mathrm{~g}: 2.0 \mathrm{~g}$ ), and reaction period (RP) (5 or 10 minutes).

A $60 \mathrm{~mL}$ of polypropylene bottle was filled with $50 \mathrm{~mL}$ of arsenic (III) working standard solution. To the solution, desired amount of sulfamic acid was added and swirled before adding zinc powder and swirled again to ensure homogeneity of the mixture. The arsine sensor paper was then inserted inside the cap of the bottle before close the bottle with cap. The bottle was swirled gently before stand for the selected reaction period. Each experiment was performed in duplicates at $25^{\circ} \mathrm{C}$ and at the levels as presented in Table 1. As soon as the reaction period was over, the 
colored arsine sensor paper was removed from the cap and used for image analysis.

\section{Color image processing}

For each colored arsine sensor paper, two images were captured by a digital camera (Sony Cyber-shot, DSC-W610) at the distance of $15 \mathrm{~cm}$. All conditions including distance, lighting conditions (automatic mode) and camera setting were kept constant for all experiments. The color (red, green and blue) of the images were transformed into digital readings from 0 to 225 using Image $\mathrm{J}$ software and used for further statistical analysis.

\section{Statistical Analysis}

To determine significant factors, Analysis of Variance (ANOVA), Student's t-analysis, correlation between response variables, linear regression analysis were carried out. Main and interaction effects plots were also formed for each color value. All these data analysis was performed using Minitab software (version 17.0) (USA). Besides that, normal probability and residual versus fitted value plots were also formed using the software.

\section{Optimisation of detection}

Optimisation plot was constructed to suggest the optimum conditions of arsenic (III) detection using the Minitab software. Validation of the suggested optimum conditions was performed by conducting the detection experiments at the suggested conditions in 5 replications. The experiments was carried out similar to the procedure as mentioned in the section of Colored complex formation at the suggested optimum conditions.

\section{Data Analysis}

To determine the significant factors that affect the detection of arsenic (III), all data analysis including linear regression analysis, Analysis of Variance (ANOVA), Student's t-analysis and correlation between response variables were implemented using the Minitab software version 17.0 (Minitab Inc., PA, USA). Main effects plot was also developed for each color value for significant contribution factors.

\section{RESULTS AND DISCUSSION}

Colorimetric method used in this work was based on modification of the methods developed by Cherukuri and Anjaneyulu (2005) ${ }^{10}$ and later by Ong et al. (2015) ${ }^{11}$.

Red, green and blue (RGB) color values are the responses in this work which produced by a change in the level of a factor. The regression analysis of red, green and blue color values are displayed in Table 3, 4 and 5, respectively. The results revealed that the main effects of mass ratio of sulfamic acid to zinc powder (MSZ) and reaction period (RP) were significant at a $5 \%$ of probability level $(P<0.05)$ for all color values. However, for interaction effect, drying weight-drying period of silver nitrate-impregnated filter-paper-reaction period (DW $x$ DP $x$ RP) interaction and drying weight-mass ratio of sulfamic acid to zinc powder -reaction period (DW $x$ MSZ x RP) interaction were significant at a $5 \%$ of probability level $(P<0.05)$ for red and green. However, such interaction effects do not exist in blue color.

Equations 1, 2 and 3 indicate the models that relate the levels of parameter and red, green and blue color values, respectively.

Red color value $=103.04+0.910 X_{1}+0.899 X_{2}$ $-3.803 X_{3}-8.053 X_{4}-1.046 X_{1} X_{2}+0.116 X_{1} X_{3}+$ $0.048 X_{1} X_{4}+0.116 X_{2} X_{3}+0.517 X_{2} X_{4}-0.500 X_{3} X_{4}$ $+0.530 X_{1} X_{2} X_{3}+2.266 X_{1} X_{2} X_{4}-1.991 X_{1} X_{3} X_{4}+$

Table 1: Low and High Levels of Factors

\begin{tabular}{lcc}
\hline Factor & $\begin{array}{c}\text { Low } \\
\text { level(-1) }\end{array}$ & $\begin{array}{c}\text { High } \\
\text { level }(+\mathbf{1})\end{array}$ \\
\hline Weight load used for drying silver nitrate-impregnated filter paper $(\mathrm{DW})\left(\mathrm{X}_{1}\right), \mathrm{g}$ & 100 & 500 \\
Reaction period $(\mathrm{RP})\left(\mathrm{X}_{4}\right)$, min. & 5 & 10 \\
Mass ratio sulfamic acid to zinc powder $(\mathrm{MSZ})\left(\mathrm{X}_{3}\right)$ & $1: 0.5$ & $4: 2$ \\
Drying period of silver nitrate-impregnated filter paper $(\mathrm{DP})\left(\mathrm{X}_{2}\right), \mathrm{s}$ & 20 & 60 \\
\hline
\end{tabular}


Table 2: Experimental results for detection of arsenic (III)

\begin{tabular}{|c|c|c|c|c|c|c|c|c|c|c|}
\hline $\begin{array}{l}\text { DW } \\
\text { (g) }\end{array}$ & $\begin{array}{l}\text { DP } \\
\text { (s) }\end{array}$ & MSZ & $\begin{array}{c}\text { RP } \\
(\min )\end{array}$ & $\begin{array}{c}\text { Arsenic (III) } \\
\text { Concentration } \\
(\mu \mathrm{g} / \mathrm{L})\end{array}$ & Red & $\begin{array}{c}\text { Color value } \\
\text { Green }\end{array}$ & Blue & $\begin{array}{l}\text { Std } \\
\text { Order }\end{array}$ & $\begin{array}{l}\text { Run } \\
\text { Order }\end{array}$ & $\begin{array}{c}\text { Center } \\
\text { Pt }\end{array}$ \\
\hline 100 & 20 & 1 & 5 & 0 & 134.929 & 153.018 & 147.315 & 1 & 1 & 1 \\
\hline 100 & 20 & 1 & 5 & 10 & 133.998 & 151.394 & 145.087 & 2 & 2 & 1 \\
\hline 100 & 20 & 1 & 5 & 50 & 132.183 & 149.634 & 141.730 & 3 & 3 & 1 \\
\hline 100 & 20 & 1 & 5 & 100 & 114.965 & 117.107 & 83.968 & 4 & 4 & 1 \\
\hline 100 & 20 & 1 & 5 & 200 & 81.996 & 75.166 & 37.681 & 5 & 5 & 1 \\
\hline 100 & 20 & 1 & 5 & 300 & 69.744 & 60.594 & 29.444 & 6 & 6 & 1 \\
\hline 100 & 20 & 4 & 5 & 0 & 130.952 & 149.481 & 141.914 & 7 & 7 & 1 \\
\hline 100 & 20 & 4 & 5 & 10 & 129.994 & 148.549 & 139.079 & 8 & 8 & 1 \\
\hline 100 & 20 & 4 & 5 & 50 & 128.233 & 142.955 & 119.901 & 9 & 9 & 1 \\
\hline 100 & 20 & 4 & 5 & 100 & 109.984 & 107.052 & 54.206 & 10 & 10 & 1 \\
\hline 100 & 20 & 4 & 5 & 200 & 76.035 & 66.674 & 39.770 & 11 & 11 & 1 \\
\hline 100 & 20 & 4 & 5 & 300 & 62.758 & 54.816 & 25.165 & 12 & 12 & 1 \\
\hline 100 & 20 & 1 & 10 & 0 & 127.595 & 146.614 & 140.348 & 13 & 13 & 1 \\
\hline 100 & 20 & 1 & 10 & 10 & 126.131 & 143.815 & 136.440 & 14 & 14 & 1 \\
\hline 100 & 20 & 1 & 10 & 50 & 124.349 & 139.430 & 125.608 & 15 & 15 & 1 \\
\hline 100 & 20 & 1 & 10 & 100 & 94.167 & 90.073 & 46.823 & 16 & 16 & 1 \\
\hline 100 & 20 & 1 & 10 & 200 & 66.920 & 57.250 & 27.520 & 17 & 17 & 1 \\
\hline 100 & 20 & 1 & 10 & 300 & 55.009 & 47.087 & 26.081 & 18 & 18 & 1 \\
\hline 100 & 20 & 4 & 10 & 0 & 127.838 & 145.430 & 139.492 & 19 & 19 & 1 \\
\hline 100 & 20 & 4 & 10 & 10 & 124.303 & 140.702 & 130.960 & 20 & 20 & 1 \\
\hline 100 & 20 & 4 & 10 & 50 & 116.619 & 127.183 & 88.124 & 21 & 21 & 1 \\
\hline 100 & 20 & 4 & 10 & 100 & 81.522 & 79.398 & 31.002 & 22 & 22 & 1 \\
\hline 100 & 20 & 4 & 10 & 200 & 63.480 & 56.726 & 23.869 & 23 & 23 & 1 \\
\hline 100 & 20 & 4 & 10 & 300 & 45.391 & 43.210 & 22.024 & 24 & 24 & 1 \\
\hline 100 & 60 & 1 & 5 & 0 & 136.792 & 153.882 & 147.563 & 25 & 25 & 1 \\
\hline 100 & 60 & 1 & 5 & 10 & 132.738 & 149.684 & 140.869 & 26 & 26 & 1 \\
\hline 100 & 60 & 1 & 5 & 50 & 129.333 & 142.622 & 127.591 & 27 & 27 & 1 \\
\hline 100 & 60 & 1 & 5 & 100 & 120.250 & 130.602 & 104.074 & 28 & 28 & 1 \\
\hline 100 & 60 & 1 & 5 & 200 & 112.361 & 111.685 & 71.303 & 29 & 29 & 1 \\
\hline 100 & 60 & 1 & 5 & 300 & 106.105 & 100.668 & 51.942 & 30 & 30 & 1 \\
\hline 100 & 60 & 4 & 5 & 0 & 127.069 & 144.288 & 133.833 & 31 & 31 & 1 \\
\hline 100 & 60 & 4 & 5 & 10 & 126.768 & 143.310 & 131.752 & 32 & 32 & 1 \\
\hline 100 & 60 & 4 & 5 & 50 & 128.274 & 144.533 & 127.201 & 33 & 33 & 1 \\
\hline 100 & 60 & 4 & 5 & 100 & 111.125 & 115.634 & 66.637 & 34 & 34 & 1 \\
\hline 100 & 60 & 4 & 5 & 200 & 92.451 & 81.186 & 44.570 & 35 & 35 & 1 \\
\hline 100 & 60 & 4 & 5 & 300 & 71.154 & 59.406 & 30.447 & 36 & 36 & 1 \\
\hline 100 & 60 & 1 & 10 & 0 & 133.098 & 152.654 & 148.877 & 37 & 37 & 1 \\
\hline 100 & 60 & 1 & 10 & 10 & 126.638 & 145.583 & 142.822 & 38 & 38 & 1 \\
\hline 100 & 60 & 1 & 10 & 50 & 112.648 & 129.302 & 127.987 & 39 & 39 & 1 \\
\hline 100 & 60 & 1 & 10 & 100 & 95.679 & 89.897 & 45.293 & 40 & 40 & 1 \\
\hline 100 & 60 & 1 & 10 & 200 & 63.582 & 55.871 & 24.001 & 41 & 41 & 1 \\
\hline 100 & 60 & 1 & 10 & 300 & 49.367 & 42.740 & 22.007 & 42 & 42 & 1 \\
\hline 100 & 60 & 4 & 10 & 0 & 122.400 & 139.719 & 133.587 & 43 & 43 & 1 \\
\hline 100 & 60 & 4 & 10 & 10 & 127.090 & 143.890 & 135.080 & 44 & 44 & 1 \\
\hline 100 & 60 & 4 & 10 & 50 & 121.196 & 130.382 & 89.783 & 45 & 45 & 1 \\
\hline 100 & 60 & 4 & 10 & 100 & 84.055 & 82.267 & 28.763 & 46 & 46 & 1 \\
\hline
\end{tabular}




\begin{tabular}{|c|c|c|c|c|c|c|c|c|c|c|}
\hline 100 & 60 & 4 & 10 & 200 & 68.112 & 62.123 & 21.130 & 47 & 47 & 1 \\
\hline 100 & 60 & 4 & 10 & 300 & 54.150 & 47.651 & 21.993 & 48 & 48 & 1 \\
\hline 500 & 60 & 1 & 10 & 0 & 136.793 & 155.491 & 152.644 & 49 & 49 & 1 \\
\hline 500 & 60 & 1 & 10 & 10 & 131.622 & 149.281 & 146.564 & 50 & 50 & 1 \\
\hline 500 & 60 & 1 & 10 & 50 & 135.405 & 151.637 & 146.537 & 51 & 51 & 1 \\
\hline 500 & 60 & 1 & 10 & 100 & 114.595 & 121.003 & 93.401 & 52 & 52 & 1 \\
\hline 500 & 60 & 1 & 10 & 200 & 104.155 & 98.945 & 52.794 & 53 & 53 & 1 \\
\hline 500 & 60 & 1 & 10 & 300 & 94.655 & 86.561 & 43.278 & 54 & 54 & 1 \\
\hline 500 & 60 & 1 & 5 & 0 & 133.138 & 149.563 & 141.373 & 55 & 55 & 1 \\
\hline 500 & 60 & 1 & 5 & 10 & 132.178 & 148.875 & 140.904 & 56 & 56 & 1 \\
\hline 500 & 60 & 1 & 5 & 50 & 125.615 & 139.866 & 117.701 & 57 & 57 & 1 \\
\hline 500 & 60 & 1 & 5 & 100 & 113.730 & 115.078 & 64.876 & 58 & 58 & 1 \\
\hline 500 & 60 & 1 & 5 & 200 & 101.051 & 94.947 & 39.514 & 59 & 59 & 1 \\
\hline 500 & 60 & 1 & 5 & 300 & 83.001 & 73.410 & 26.479 & 60 & 60 & 1 \\
\hline 500 & 20 & 1 & 5 & 0 & 128.106 & 145.621 & 139.839 & 61 & 61 & 1 \\
\hline 500 & 20 & 1 & 5 & 10 & 130.079 & 147.265 & 140.111 & 62 & 62 & 1 \\
\hline 500 & 20 & 1 & 5 & 50 & 130.201 & 145.643 & 129.774 & 63 & 63 & 1 \\
\hline 500 & 20 & 1 & 5 & 100 & 102.316 & 102.132 & 63.249 & 64 & 64 & 1 \\
\hline 500 & 20 & 1 & 5 & 200 & 70.654 & 61.166 & 29.168 & 65 & 65 & 1 \\
\hline 500 & 20 & 1 & 5 & 300 & 59.520 & 50.683 & 24.021 & 66 & 66 & 1 \\
\hline 500 & 20 & 4 & 5 & 0 & 131.697 & 150.428 & 147.854 & 67 & 67 & 1 \\
\hline 500 & 20 & 4 & 5 & 10 & 125.379 & 141.680 & 131.462 & 68 & 68 & 1 \\
\hline 500 & 20 & 4 & 5 & 50 & 96.648 & 106.171 & 70.494 & 69 & 69 & 1 \\
\hline 500 & 20 & 4 & 5 & 100 & 76.423 & 71.536 & 25.635 & 70 & 70 & 1 \\
\hline 500 & 20 & 4 & 5 & 200 & 49.939 & 45.328 & 22.642 & 71 & 71 & 1 \\
\hline 500 & 20 & 4 & 5 & 300 & 46.059 & 42.446 & 25.182 & 72 & 72 & 1 \\
\hline 500 & 20 & 1 & 10 & 0 & 130.418 & 148.622 & 145.224 & 73 & 73 & 1 \\
\hline 500 & 20 & 1 & 10 & 10 & 131.638 & 148.659 & 144.986 & 74 & 74 & 1 \\
\hline 500 & 20 & 1 & 10 & 50 & 126.705 & 143.390 & 138.145 & 75 & 75 & 1 \\
\hline 500 & 20 & 1 & 10 & 100 & 119.817 & 124.719 & 91.182 & 76 & 76 & 1 \\
\hline 500 & 20 & 1 & 10 & 200 & 90.857 & 88.574 & 55.037 & 77 & 77 & 1 \\
\hline 500 & 20 & 1 & 10 & 300 & 78.105 & 70.236 & 32.173 & 78 & 78 & 1 \\
\hline 500 & 20 & 4 & 10 & 0 & 128.167 & 144.262 & 137.551 & 79 & 79 & 1 \\
\hline 500 & 20 & 4 & 10 & 10 & 129.817 & 146.586 & 140.104 & 80 & 80 & 1 \\
\hline 500 & 20 & 4 & 10 & 50 & 123.058 & 136.867 & 119.304 & 81 & 81 & 1 \\
\hline 500 & 20 & 4 & 10 & 100 & 114.697 & 115.249 & 65.165 & 82 & 82 & 1 \\
\hline 500 & 20 & 4 & 10 & 200 & 89.996 & 81.248 & 30.444 & 83 & 83 & 1 \\
\hline 500 & 20 & 4 & 10 & 300 & 72.356 & 60.805 & 23.572 & 84 & 84 & 1 \\
\hline 500 & 60 & 4 & 5 & 0 & 135.358 & 153.436 & 148.180 & 85 & 85 & 1 \\
\hline 500 & 60 & 4 & 5 & 10 & 131.337 & 149.016 & 143.035 & 86 & 86 & 1 \\
\hline 500 & 60 & 4 & 5 & 50 & 127.215 & 142.622 & 128.297 & 87 & 87 & 1 \\
\hline 500 & 60 & 4 & 5 & 100 & 109.072 & 108.769 & 62.776 & 88 & 88 & 1 \\
\hline 500 & 60 & 4 & 5 & 200 & 82.429 & 69.917 & 27.049 & 89 & 89 & 1 \\
\hline 500 & 60 & 4 & 5 & 300 & 56.139 & 48.689 & 21.712 & 90 & 90 & 1 \\
\hline 500 & 60 & 4 & 10 & 0 & 132.673 & 150.651 & 143.491 & 91 & 91 & 1 \\
\hline 500 & 60 & 4 & 10 & 10 & 116.933 & 131.173 & 112.984 & 92 & 92 & 1 \\
\hline 500 & 60 & 4 & 10 & 50 & 121.582 & 124.256 & 70.959 & 93 & 93 & 1 \\
\hline 500 & 60 & 4 & 10 & 100 & 83.550 & 74.764 & 26.496 & 94 & 94 & 1 \\
\hline 500 & 60 & 4 & 10 & 200 & 66.479 & 55.596 & 19.859 & 95 & 95 & 1 \\
\hline 500 & 60 & 4 & 10 & 300 & 47.531 & 41.547 & 21.623 & 96 & 96 & 1 \\
\hline
\end{tabular}


$0.987 X_{2} X_{3} X_{4}-0.719 X_{1} X_{2} X_{3} X_{4}$

Green color value $=110.910+0.640 \mathrm{X}_{1}+0.741 \mathrm{X}_{2}$ $-4.683 X_{3}-8.836 X_{4}-1.472 X_{1} X_{2}-0.937 X_{1} X_{3}$ $-0.192 X_{1} X_{4}-0.140 X_{2} X_{3}+0.207 X_{2} X_{4}-0.130 X_{3} X_{4}+$ $0.461 X_{1} X_{2} X_{3}+2.205 X_{1} X_{2} X_{4}-2.141 X_{1} X_{3} X_{4}+0.869$ $\mathrm{X}_{2} \mathrm{X}_{3} \mathrm{X}_{4}-0.902 \mathrm{X}_{1} \mathrm{X}_{2} \mathrm{X}_{3} \mathrm{X}_{4}$
Blue color value $=86.74+0.02 \mathrm{X}_{1}+0.10 \mathrm{X}_{2}$

(1) $\quad-7.87 \mathrm{X}_{3}-8.51 \mathrm{X}_{4}-1.48 \mathrm{X}_{1} \mathrm{X}_{2}-1.16 \mathrm{X}_{1} \mathrm{X}_{3}-0.10 \mathrm{X}_{1} \mathrm{X}_{4}$ $-0.79 X_{2} X_{3}-0.51 X_{2} X_{4}-0.18 X_{3} X_{4}+0.40 X_{1} X_{2} X_{3}+$ $0.93 X_{1} X_{2} X_{4}-0.73 X_{1} X_{3} X_{4}-0.17 X_{2} X_{3} X_{4}-0.80 X_{1} X_{2} X_{3} X_{4}$

A factor that positively significant can be ...(2) seen from the color value decreases as the change

Table 3: Statistical Parameters for $2^{4}$ full factorial design of red color value

\begin{tabular}{lccccc}
\hline Term & Effects & Coefficients & Standard Error & T-value & P-value \\
\hline Constant & - & 103.04 & 1.21 & 122.11 & 0.000 \\
DW & 1.820 & 0.910 & 0.862 & 1.06 & 0.295 \\
DP & 1.798 & 0.899 & 0.862 & 1.04 & 0.301 \\
MSZ & -7.607 & -3.803 & 0.862 & -4.41 & 0.000 \\
RP & -16.106 & -8.053 & 0.862 & -9.34 & 0.000 \\
DW x DP & -2.091 & -1.046 & 0.862 & -1.21 & 0.229 \\
DW x MSZ & 0.232 & 0.116 & 0.862 & -0.80 & 0.429 \\
DW x RP & 0.096 & 0.048 & 0.862 & 0.06 & 0.956 \\
DP x MSZ & 0.232 & 0.116 & 0.862 & -0.80 & 0.429 \\
DP x RP & 1.034 & 0.517 & 0.862 & 0.60 & 0.551 \\
MSZ x RP & -1.000 & -0.500 & 0.862 & -0.58 & 0.564 \\
DW x DP x MSZ & 1.060 & 0.530 & 0.862 & 0.61 & 0.541 \\
DW x DP x RP & 4.532 & 2.266 & 0.862 & 2.63 & 0.010 \\
DW x MSZ x RP & -3.982 & -1.991 & 0.862 & -2.31 & 0.024 \\
DP x MSZ x RP & 1.974 & 0.987 & 0.862 & 1.14 & 0.256 \\
DW x DP x MSZ x RP & -1.438 & -0.719 & 0.862 & -0.83 & 0.407 \\
\hline
\end{tabular}

Table 4: Statistical Parameters for $2^{4}$ full factorial design of green color value

\begin{tabular}{lccccc}
\hline Term & Effects & Coefficients & Standard Error & T-value & P-value \\
\hline Constant & - & 110.910 & 0.950 & 116.73 & 0.000 \\
DW & 1.281 & 0.640 & 0.950 & 0.67 & 0.502 \\
DP & 1.482 & 0.741 & 0.950 & 0.78 & 0.438 \\
MSZ & -9.366 & -4.683 & 0.950 & -4.93 & 0.000 \\
RP & -17.673 & -8.836 & 0.950 & -9.30 & 0.000 \\
DW $\times$ DP & -2.944 & -1.472 & 0.950 & -1.55 & 0.125 \\
DW $\times$ MSZ & -1.874 & -0.937 & 0.950 & -0.99 & 0.327 \\
DW $\times$ RP & -0.383 & -0.192 & 0.950 & -0.20 & 0.841 \\
DP x MSZ & -0.820 & -0.410 & 0.950 & -0.43 & 0.668 \\
DP x RP & 0.414 & 0.207 & 0.950 & 0.22 & 0.828 \\
MSZ x RP & -0.260 & -0.130 & 0.950 & -0.14 & 0.892 \\
DW $\times$ DP x MSZ & 0.922 & 0.461 & 0.950 & 0.49 & 0.629 \\
DW $\times$ DP x RP & 4.410 & 2.205 & 0.950 & 2.32 & 0.023 \\
DW x MSZ x RP & -4.283 & -2.141 & 0.950 & -2.25 & 0.027 \\
DP x MSZ x RP & 1.739 & 0.869 & 0.950 & 0.92 & 0.363 \\
DW x DP x MSZ x RP & -1.803 & -0.902 & 0.950 & -0.95 & 0.346 \\
\hline
\end{tabular}


from low to high level or vice versa, while if the colors are red, green and blue formed a high level of the same factors, it is negative effect. Figures 1, 2 and 3 illustrate the main effects of the factors for red, green and blue color values.

Tables 6,7 and 8 show the results of Analysis of Variance for three response colors, respectively. The sum of squares used to estimate the factors' effects and F-ratios are also presented in the tables. The results revealed that the main effects of MSZ and RP are highly significant (at $5 \%$ level of significance). However, the MSZ and RP interaction are not significant and most of the interaction effects are insignificant as compared to other effects accepts for DW x DP x RP and DW x MSZ x RP. Therefore, recalculation of regression coefficients, standard error, $t$ and $p$-values were conducted and the results are shown in Tables 9, 10 and 11 for red, green and blue color values, respectively.

In equations 4, 5 and 6, respectively, shows a reduced model equation with resultant coefficients for red, green and blue color values.

Red color value $=103.04-3.803 X_{3}-8.053 X_{4}+$ $2.266 \mathrm{X}_{1} \mathrm{X}_{2} \mathrm{X}_{4}-1.991 \mathrm{X}_{1} \mathrm{X}_{3} \mathrm{X}_{4}$
Green color value $=110.910-4.683 X_{3}-8.836 X_{4}+$ $2.205 \mathrm{X}_{1} \mathrm{X}_{2} \mathrm{X}_{4}-2.141 \mathrm{X}_{1} \mathrm{X}_{3} \mathrm{X}_{4}$ Blue color value $=86.74-7.87 \mathrm{X}_{3}-8.51 \mathrm{X}_{4}$

Table 12, 13 and 14 illustrate the output following the removal of the insignificant main effects and interactions. The results of ANOVA for reduced models of red, green and blue color values are shown in Table 12, 13 and 14, respectively. From the results, we have sufficient evidence to conclude that reaction period (RP) was the strongest effect of the overall contributed to the three color intensities. The reduced model now contains only the main effects MSZ, RP and the DW $x$ DP $x$ RP and DW $x$ MSZ $x$ $\mathrm{RP}$ interactions. The $\mathrm{X}_{4}$ coefficient was found to be the largest negative coefficient for the three models (5), (6) and (7), showing that the longer the reaction period, three color values decreased accordingly. The mass ratio of sulfamic acid to zinc powder (MSZ) was the second important factor. Third and fourth significant factors which significantly contributed for red and green color values were drying weightdrying period of silver nitrate-impregnated filterpaper-reaction period (DW x DP x RP) interaction and drying weight-mass ratio of sulfamic acid to zinc powder -reaction period (DW $x$ MSZ $x$ RP) interaction, respectively.

Table 5: Statistical Parameters for $2^{4}$ full factorial design of blue color value

\begin{tabular}{|c|c|c|c|c|c|}
\hline Term & Effects & Coefficients & Standard Error & T-value & P-value \\
\hline Constant & & 86.74 & 1.18 & 73.81 & 0.000 \\
\hline DW & 0.05 & 0.02 & 1.18 & 0.02 & 0.985 \\
\hline $\mathrm{DP}$ & 0.20 & 0.10 & 1.18 & 0.08 & 0.934 \\
\hline MSZ & -15.74 & -7.87 & 1.18 & -6.70 & 0.000 \\
\hline $\mathrm{RP}$ & -17.01 & -8.51 & 1.18 & -7.24 & 0.000 \\
\hline DW x DP & -2.95 & -1.48 & 1.18 & -1.26 & 0.213 \\
\hline DW $\times$ MSZ & -2.32 & -1.16 & 1.18 & -0.99 & 0.327 \\
\hline $\mathrm{DW} \times \mathrm{RP}$ & -0.20 & -0.10 & 1.18 & -0.09 & 0.932 \\
\hline DP $\times$ MSZ & -1.58 & -0.79 & 1.18 & -0.67 & 0.503 \\
\hline $\mathrm{DP} \times \mathrm{RP}$ & -1.03 & -0.51 & 1.18 & -0.44 & 0.664 \\
\hline MSZ x RP & -0.36 & -0.18 & 1.18 & -0.15 & 0.877 \\
\hline $\mathrm{DW} \times \mathrm{DP} \times \mathrm{MSZ}$ & 0.79 & 0.40 & 1.18 & 0.34 & 0.737 \\
\hline $\mathrm{DW} \times \mathrm{DP} \times \mathrm{RP}$ & 1.87 & 0.93 & 1.18 & 0.80 & 0.429 \\
\hline $\mathrm{DW} \times \mathrm{MSZ} \times \mathrm{RP}$ & -1.45 & -0.73 & 1.18 & -0.62 & 0.539 \\
\hline DP $\times$ MSZ $\times$ RP & -0.34 & -0.17 & 1.18 & -0.14 & 0.887 \\
\hline $\mathrm{DW} \times \mathrm{DP} \times \mathrm{MSZ} \times \mathrm{RP}$ & -1.60 & -0.80 & 1.18 & -0.68 & 0.498 \\
\hline
\end{tabular}


Table 6: Analysis of Variance (ANOVA) for red color value

\begin{tabular}{|c|c|c|c|c|c|}
\hline Term & $\begin{array}{l}\text { Degrees } \\
\text { of Freedom }\end{array}$ & $\begin{array}{c}\text { Sum of } \\
\text { Squares (SS) }\end{array}$ & $\begin{array}{c}\text { Mean } \\
\text { Square (MS) }\end{array}$ & F-value & P-value \\
\hline BLOCKS & 6 & - & - & - & - \\
\hline DW & 1 & 79.5 & 79.5 & 1.11 & 0.295 \\
\hline DP & 1 & 77.6 & 77.6 & 1.09 & 0.301 \\
\hline MSZ & 1 & 1388.6 & 1388.6 & 19.45 & 0.000 \\
\hline $\mathrm{RP}$ & 1 & 6226.0 & 6226.0 & 87.18 & 0.000 \\
\hline $\mathrm{DW} \times \mathrm{DP}$ & 1 & 104.9 & 104.9 & 1.47 & 0.229 \\
\hline DW $\times$ MSZ & 1 & 45.2 & 45.2 & 0.63 & 0.429 \\
\hline $\mathrm{DW} \times \mathrm{RP}$ & 1 & 0.2 & 0.2 & 0.00 & 0.956 \\
\hline DP x MSZ & 1 & 1.3 & 1.3 & 0.02 & 0.894 \\
\hline $\mathrm{DP} \times \mathrm{RP}$ & 1 & 25.7 & 25.7 & 0.36 & 0.551 \\
\hline MSZ x RP & 1 & 24.0 & 24.0 & 0.34 & 0.564 \\
\hline $\mathrm{DW} \times \mathrm{DP} \times \mathrm{MSZ}$ & 1 & 26.9 & 26.9 & 0.38 & 0.541 \\
\hline DW x DP x RP & 1 & 492.9 & 492.9 & 6.90 & 0.010 \\
\hline $\mathrm{DW} \times \mathrm{MSZ} \times \mathrm{RP}$ & 1 & 380.5 & 380.5 & 5.33 & 0.024 \\
\hline $\mathrm{DP} \times \mathrm{MSZ} \times \mathrm{RP}$ & 1 & 93.5 & 93.5 & 1.31 & 0.256 \\
\hline DW $x$ DT $x$ MSZ $x$ RP & 1 & 49.6 & 49.6 & 0.69 & 0.407 \\
\hline Error & 75 & 5355.9 & 71.4 & - & - \\
\hline Total & 96 & 74467.4 & - & - & - \\
\hline
\end{tabular}

$S=8.45055 \quad R-s q=92.81 \% \quad R-s q(a d j)=90.89 \%$

Table 7. Analysis of Variance (ANOVA) for green color value

\begin{tabular}{lccccc}
\hline Term & $\begin{array}{c}\text { Degrees } \\
\text { of Freedom }\end{array}$ & $\begin{array}{c}\text { Sum of } \\
\text { Squares (SS) }\end{array}$ & $\begin{array}{c}\text { Mean } \\
\text { Square (MS) }\end{array}$ & F-value & P-value \\
\hline BLOCKS & 6 & - & - & - & \\
DW & 1 & 39 & 39.4 & 0.45 & 0.502 \\
DP & 1 & 53 & 52.7 & 0.61 & 0.438 \\
MSZ & 1 & 2105 & 2105.2 & 24.29 & 0.000 \\
RP & 1 & 7496 & 7495.7 & 86.49 & 0.000 \\
DW $\times$ DP & 1 & 208 & 208.1 & 2.40 & 0.125 \\
DW $\times$ MSZ & 1 & 84 & 84.3 & 0.97 & 0.327 \\
DW $\times$ RP & 1 & 4 & 3.5 & 0.04 & 0.841 \\
DP $\times$ MSZ & 1 & 16 & 16.1 & 0.19 & 0.668 \\
DP x RP & 1 & 4 & 4.1 & 0.05 & 0.828 \\
MSZ $\times$ RP & 1 & 2 & 1.6 & 0.02 & 0.892 \\
DW $\times$ DP $\times$ MSZ & 1 & 20 & 20.4 & 0.24 & 0.629 \\
DW $\times$ DP $\times$ RP & 1 & 467 & 466.7 & 5.39 & 0.023 \\
DW $\times$ MSZ x RP & 1 & 440 & 440.2 & 5.08 & 0.027 \\
DP $\times$ MSZ $\times$ RP & 1 & 73 & 72.6 & 0.84 & 0.363 \\
DW $\times$ DP $\times$ MSZ $\times$ RP & 1 & 78 & 78.0 & 0.90 & 0.346 \\
Error & 75 & 6500 & 86.7 & - & - \\
Total & 96 & 140097 & - & - & - \\
\hline
\end{tabular}

$S=9.30933 R-s q=95.36 \% R-s q(\operatorname{adj})=94.12 \%$ 
Table 8: Analysis of Variance (ANOVA) for blue color value

\begin{tabular}{|c|c|c|c|c|c|}
\hline Term & $\begin{array}{l}\text { Degrees } \\
\text { of Freedom }\end{array}$ & $\begin{array}{c}\text { Sum of } \\
\text { Squares (SS) }\end{array}$ & $\begin{array}{c}\text { Mean } \\
\text { Square (MS) }\end{array}$ & F-value & P-value \\
\hline BLOCKS & 6 & - & - & - & - \\
\hline DW & 1 & 0 & 0.1 & 0.00 & 0.985 \\
\hline $\mathrm{DP}$ & 1 & 1 & 0.9 & 0.01 & 0.934 \\
\hline MSZ & 1 & 5947 & 5947.2 & 44.85 & 0.000 \\
\hline $\mathrm{RP}$ & 1 & 6944 & 6944.2 & 52.37 & 0.000 \\
\hline $\mathrm{DW} \times \mathrm{DP}$ & 1 & 209 & 209.2 & 1.58 & 0.213 \\
\hline $\mathrm{DW} \times \mathrm{MSZ}$ & 1 & 129 & 129.3 & 0.98 & 0.327 \\
\hline $\mathrm{DW} \times \mathrm{RP}$ & 1 & 1 & 1.0 & 0.01 & 0.932 \\
\hline DT $x$ MSZ & 1 & 60 & 60.2 & 0.45 & 0.503 \\
\hline $\mathrm{DT} \times \mathrm{RP}$ & 1 & 25 & 25.3 & 0.19 & 0.664 \\
\hline MSZ x RP & 1 & 3 & 3.2 & 0.02 & 0.877 \\
\hline $\mathrm{DW} \times \mathrm{DP} \times \mathrm{MSZ}$ & 1 & 15 & 15.1 & 0.11 & 0.737 \\
\hline $\mathrm{DW} \times \mathrm{DP} \times \mathrm{RP}$ & 1 & 84 & 83.8 & 0.63 & 0.429 \\
\hline $\mathrm{DW} \times \mathrm{MSZ} \times \mathrm{RP}$ & 1 & 51 & 50.5 & 0.38 & 0.539 \\
\hline $\mathrm{DP} \times \mathrm{MSZ} \times \mathrm{RP}$ & 1 & 3 & 2.7 & 0.02 & 0.887 \\
\hline$D W \times D P \times M S Z \times R P$ & 1 & 62 & 61.6 & 0.46 & 0.498 \\
\hline Error & 75 & 9946 & 132.6 & - & - \\
\hline Total & 96 & 239449 & - & - & - \\
\hline
\end{tabular}

Table 9: Statistical parameters for $2^{4}$ full factorial design of red color value for reduced model

\begin{tabular}{lccccc}
\hline Term & Effects & Coefficients & Standard Error & T-value & P-value \\
\hline Constant & - & 103.04 & 0.844 & 124.75 & 0.000 \\
MSZ & -7.607 & -3.803 & 0.844 & 4.50 & 0.000 \\
RP & -16.106 & -8.053 & 0.844 & -9.54 & 0.000 \\
DW x DP x RP & 4.532 & 2.266 & 0.844 & 2.68 & 0.009 \\
DW x MSZ x RP & -3.982 & -1.991 & 0.844 & -2.36 & 0.021 \\
\hline
\end{tabular}

Table 10: Statistical parameters for $2^{4}$ full factorial design of green color value for reduced model

\begin{tabular}{lccccc}
\hline Term & Effects & Coefficients & Standard Error & T-value & P-value \\
\hline Constant & - & 110.910 & 0.926 & 119.76 & 0.000 \\
MSZ & -9.366 & -4.683 & 0.926 & -5.06 & 0.000 \\
RP & -17.673 & -8.836 & 0.926 & -9.54 & 0.000 \\
DW x DP x RP & 4.410 & 2.205 & 0.926 & 2.38 & 0.019 \\
DW x MSZ x RP & -4.283 & -2.141 & 0.926 & -2.31 & 0.023 \\
\hline
\end{tabular}


Table 11: Statistical parameters for $2^{4}$ full factorial design of blue color value for reduced model

\begin{tabular}{lccccc}
\hline Term & Effects & Coefficients & Standard Error & T-value & P-value \\
\hline Constant & & 86.74 & 1.12 & 77.48 & 0.000 \\
MSZ & -15.74 & -7.87 & 1.12 & -7.03 & 0.000 \\
RP & -17.01 & -8.51 & 1.12 & -7.60 & 0.000 \\
\hline
\end{tabular}

Table 12: Analysis of Variance (ANOVA) of Red Color Value for reduced model

\begin{tabular}{lccccc}
\hline Term & $\begin{array}{c}\text { Degrees } \\
\text { of Freedom }\end{array}$ & $\begin{array}{c}\text { Sum of } \\
\text { Squares (SS) }\end{array}$ & $\begin{array}{c}\text { Mean } \\
\text { Square (MS) }\end{array}$ & F & P \\
\hline BLOCKS & 6 & - & - & - & - \\
MSZ & 1 & 1388.6 & 1388.6 & 20.29 & 0.000 \\
RP & 1 & 6226.0 & 6226.0 & 90.99 & 0.000 \\
DW x DP x RP & 1 & 492.9 & 492.9 & 7.20 & 0.009 \\
DW x MSZ x RP & 1 & 380.5 & 380.5 & 5.56 & 0.021 \\
Error & 87 & 5884.3 & 68.4 & - & - \\
\hline
\end{tabular}

$S=8.27180 R-s q=92.10 \% \quad R-s q(a d j)=91.27 \%$

Table 13: Analysis of Variance (ANOVA) of Green Color Value for reduced model

\begin{tabular}{lccccc}
\hline Term & $\begin{array}{c}\text { Degrees } \\
\text { of Freedom }\end{array}$ & $\begin{array}{c}\text { Sum of } \\
\text { Squares (SS) }\end{array}$ & $\begin{array}{c}\text { Mean } \\
\text { Square (MS) }\end{array}$ & $\mathbf{F}$ & $\mathbf{P}$ \\
\hline BLOCKS & 6 & - & - & - & - \\
MSZ & 1 & 2105 & 2105.2 & 25.57 & 0.000 \\
RP & 1 & 7496 & 7495.7 & 91.04 & 0.000 \\
DW x DP x RP & 1 & 467 & 466.7 & 5.67 & 0.019 \\
DW x MSZ x RP & 1 & 440 & 440.2 & 5.35 & 0.023 \\
Error & 87 & 7081 & 82.3 & - & - \\
\hline
\end{tabular}

$S=9.07367 R-s q=94.95 \% \quad R-s q(\operatorname{adj})=94.42 \%$

Table 14: Analysis of Variance (ANOVA) of Blue Color Value for reduced model

\begin{tabular}{lccccc}
\hline Term & $\begin{array}{c}\text { Degrees } \\
\text { of Freedom }\end{array}$ & $\begin{array}{c}\text { Sum of } \\
\text { Squares (SS) }\end{array}$ & $\begin{array}{c}\text { Mean } \\
\text { Square (MS) }\end{array}$ & F & P \\
\hline BLOCKS & 6 & - & - & - & - \\
MSZ & 1 & 5947 & 5947.2 & 49.43 & 0.000 \\
RP & 1 & 6944 & 6944.2 & 57.71 & 0.000 \\
Error & 88 & 10589 & 120.3 & - & - \\
Total & 96 & 239449 & - & - & - \\
BLOCKS & 6 & - & - & - & - \\
\hline S $=10.9693$ & R-sq $=95.58 \%$ & R-sq(adj) $=95.23 \%$ & &
\end{tabular}


Figures 4, 5 and 6 present the interaction effects of red, green and blue color values, respectively. It is evident that, the effects of both MSZ and RP were more observable at high levels for all color as shown in the interaction plots of Figures 4, 5 and 6.

\section{Main Effects Plot for RED}

Fitted Means

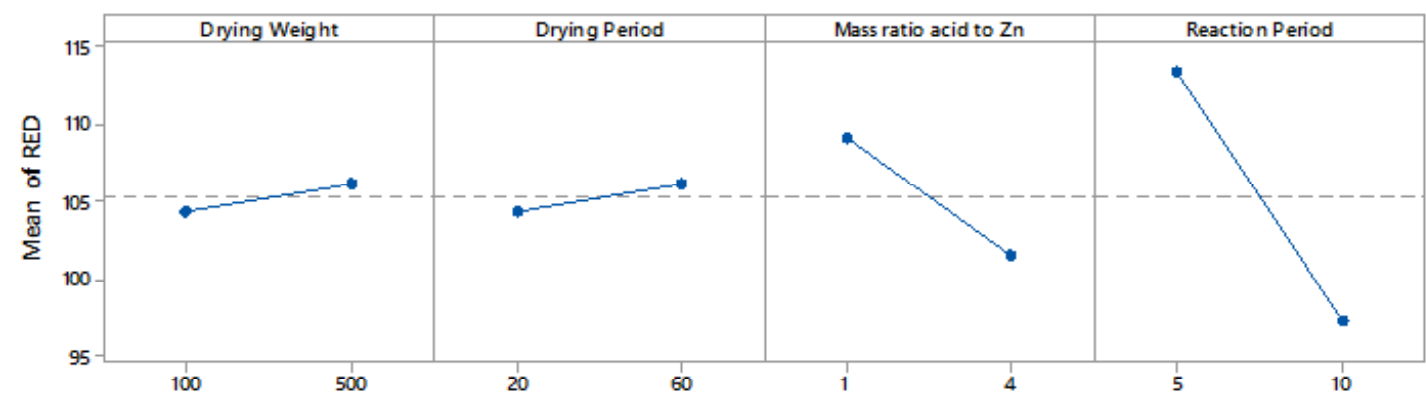

Fig. 1: Main effects plot for Red Color Value

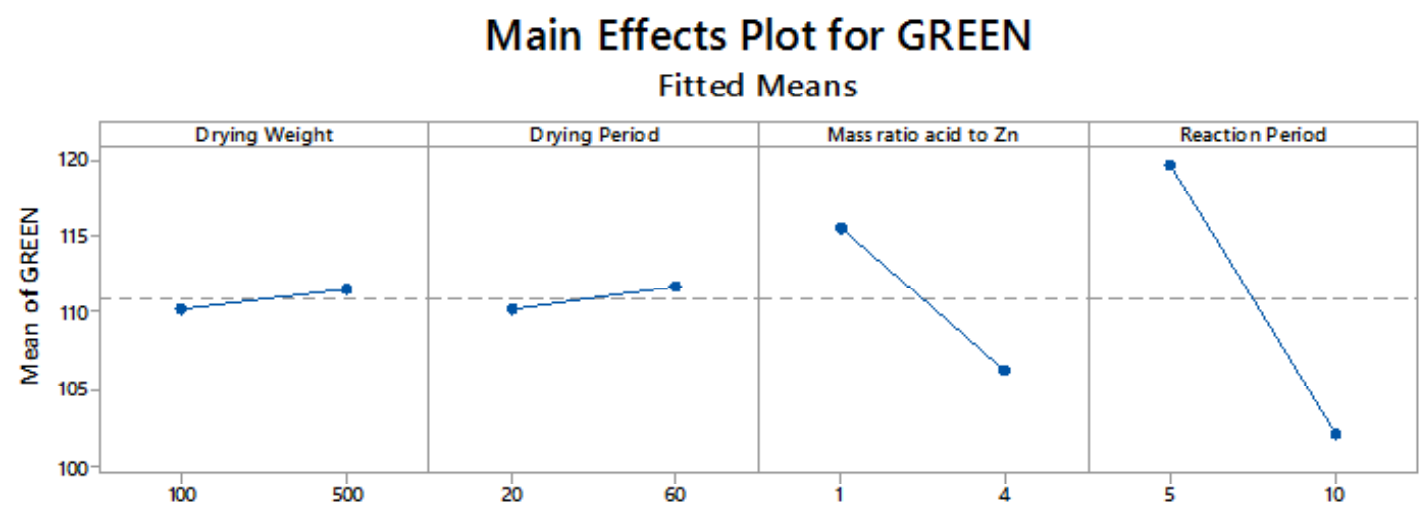

Fig. 2: Main effects plot for Green Color Value

Main Effects Plot for BLUE

Fitted Means

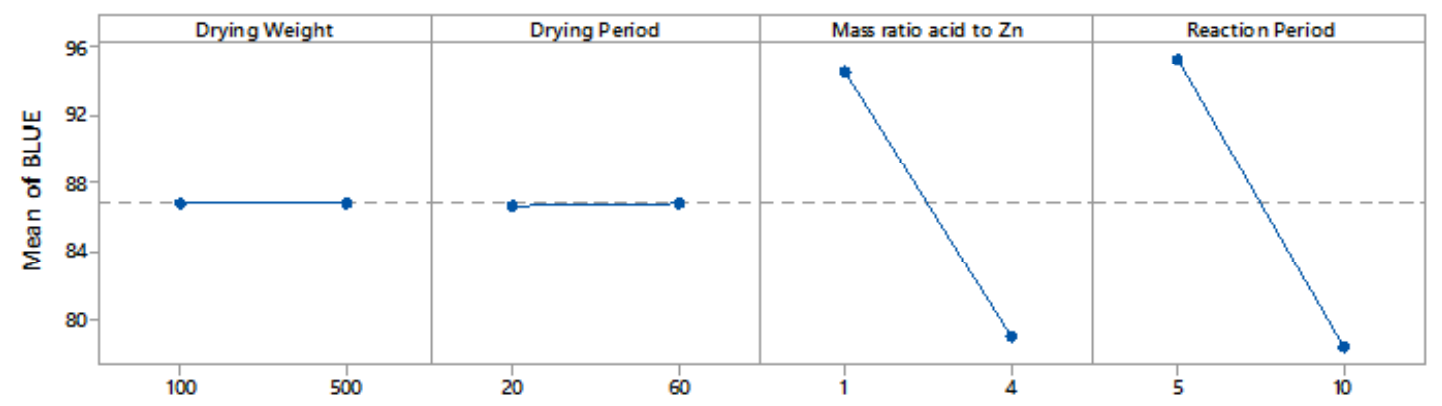

Fig. 3: Main effects plot for Blue Color Value 


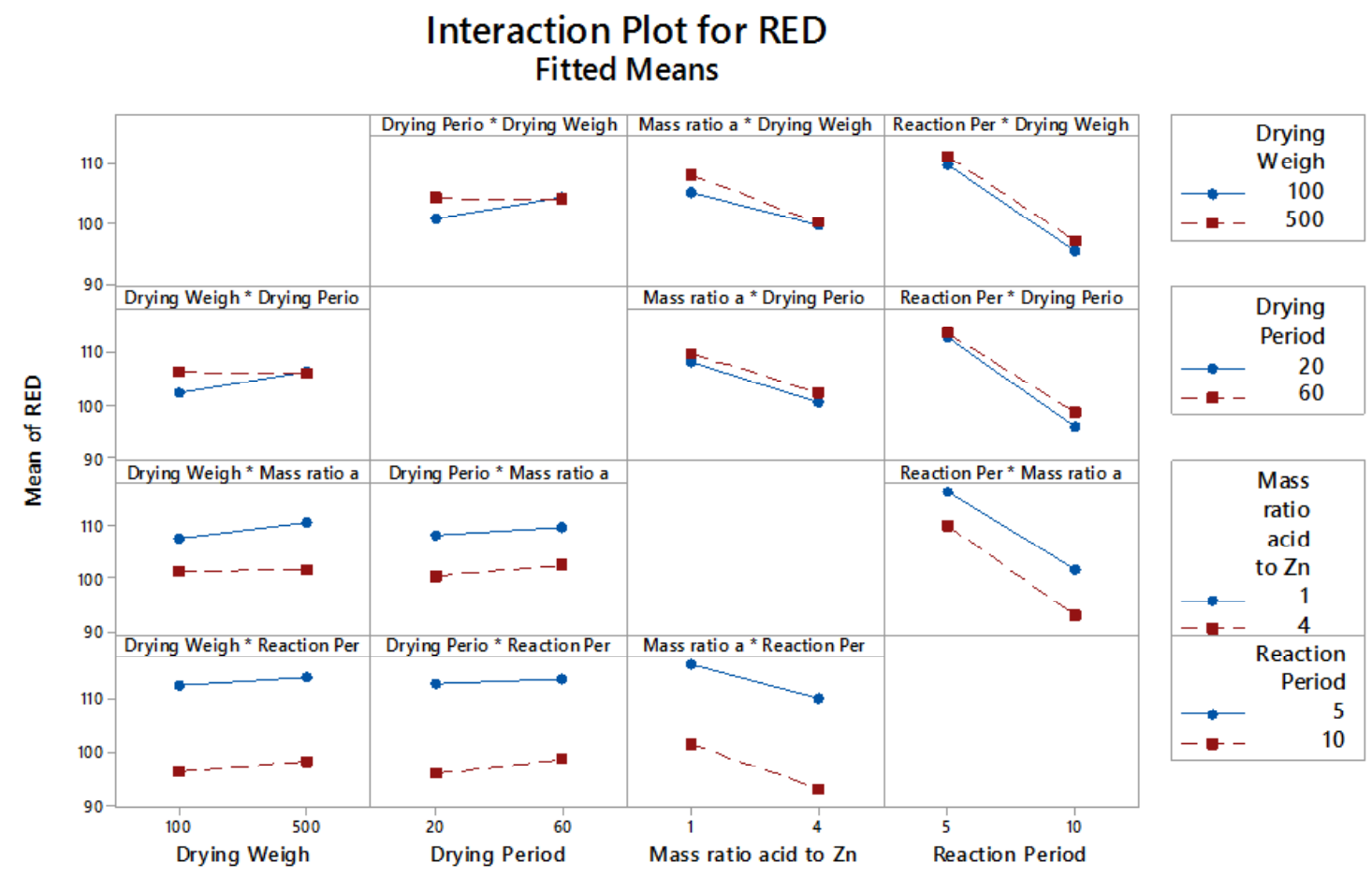

Fig. 4: Interaction effects of reduced model for Red Color Value

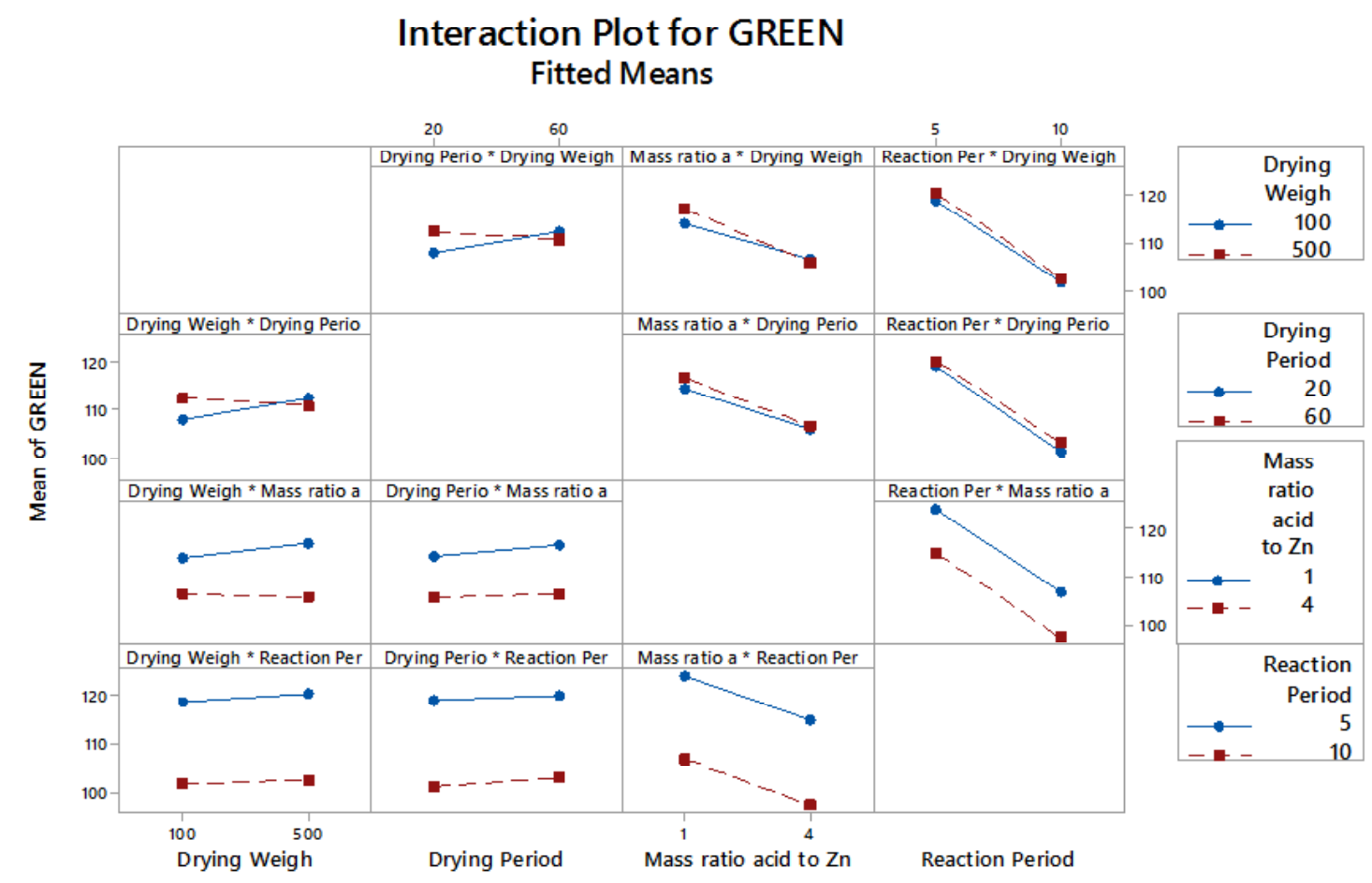

Fig. 5: Interaction effects for Green Color Value 


\section{Normal distribution plot}

The estimate values for all response colors showed that the experimental data are normally distributed as the experimental points were reasonably aligned, as shown in Figures 7, 8 and 9 of the normal probability plots of residual values. The residual plots showed outliers are occurred (Fig. 10, 11 and 12). However, the results showed that there were no outlier between the ranges of +25 to -15 for red color value while the ranges for green and blue color values are between +25 to -20 and +25 to -30 respectively.

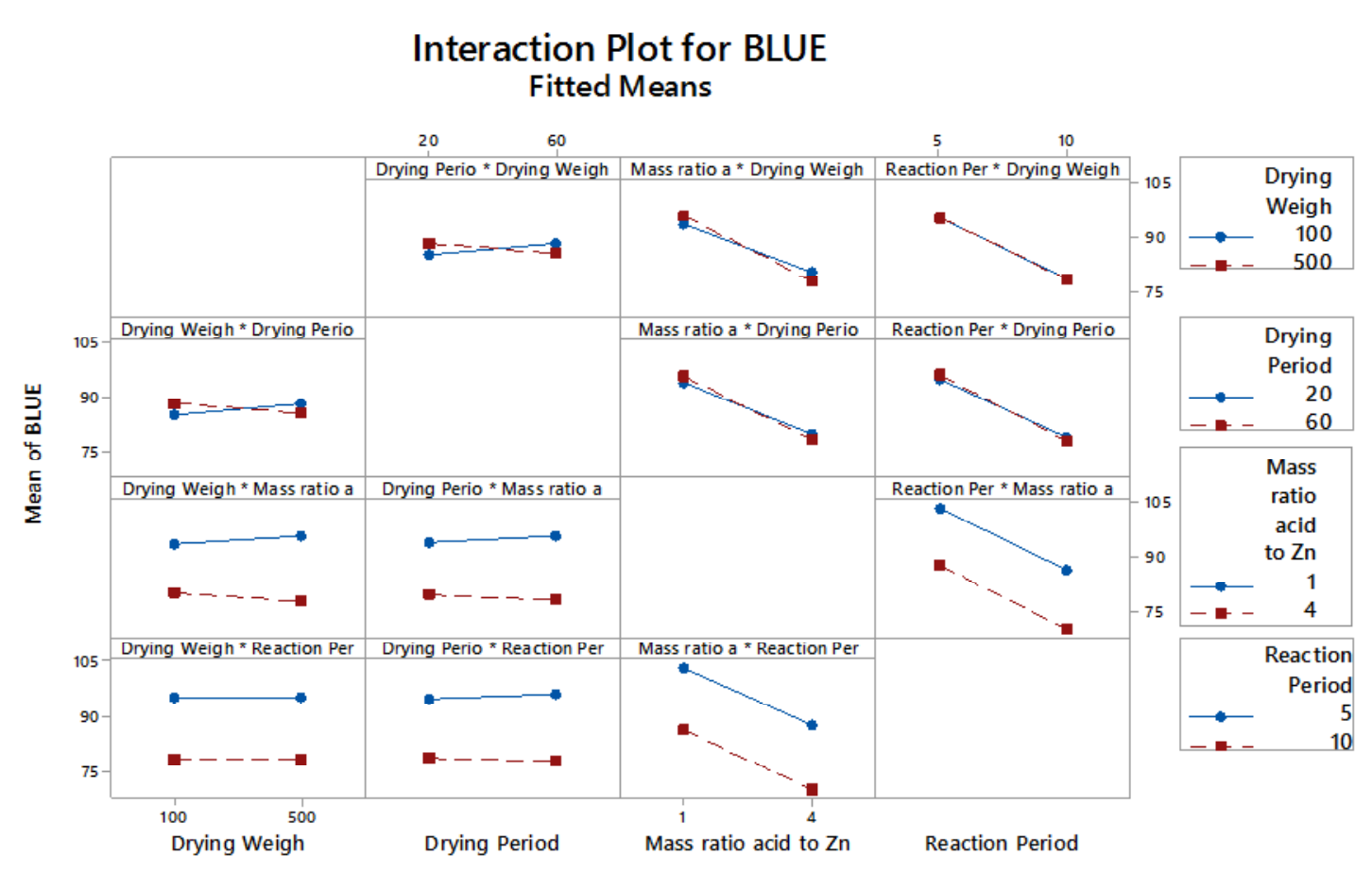

Fig. 6: Interaction effects for Blue Color Value

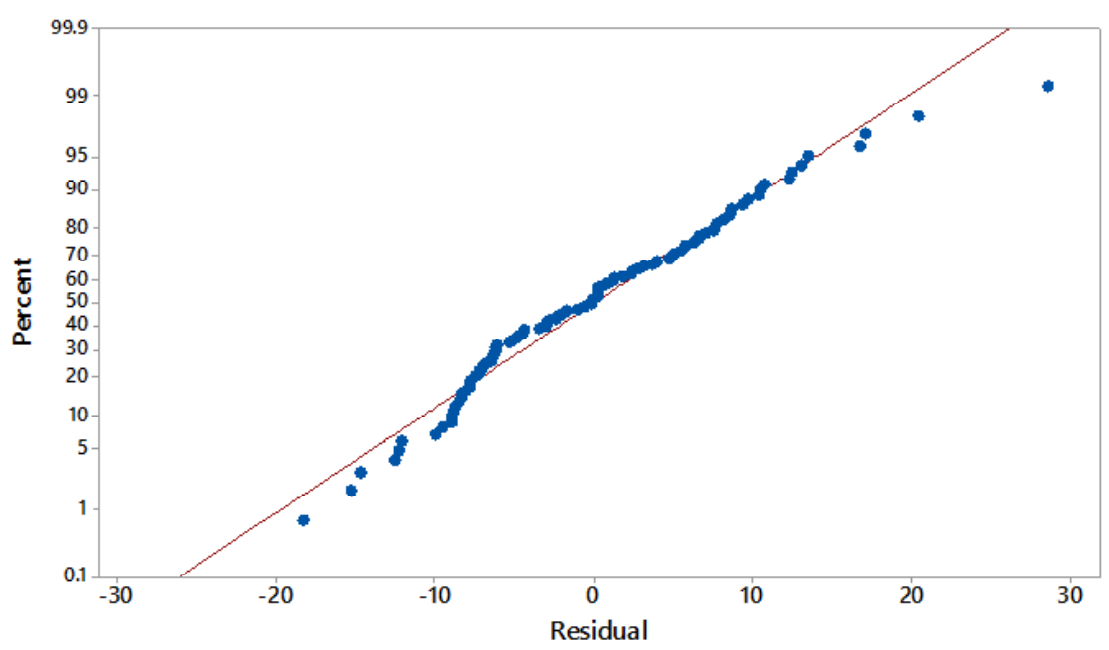

Fig. 7: Normal probability plot of residual values for red color value 


\section{Main effect of reaction period}

Figures 1 to 3 show that red, green and blue values decreased by $15.79 \%, 14.29 \%$ and $17.89 \%$, respectively, when the reaction period increased from 5 minutes to 10 minutes. The results from Tables 9 to 11 also exhibited that the reaction period also plays a significant role on color values. This can be explained by the fact that more arsenic (III) is reduced to arsine gas which will react with silver ions and produce darker color compound on the impregnated filter paper when longer reaction period was used.

\section{Main effect of mass ratio of sulfamic acid to} zinc powder

As it can be seen from Figures 4 to 6 , the mass ratio of sulfamic acid to zinc powder is the most significant factor as indicated by an increase in the mass ratio of sulfamic acid to zinc powder from $1 \mathrm{~g}: 0.5 \mathrm{~g}$ to $4 \mathrm{~g}: 2 \mathrm{~g}$, caused decrease in the RGB values with the highest decrease in color values of $17.02 \%, 7.83 \%, 6.42 \%$, for blue, green and red color values, respectively, as shown in Figures 1 to 3 . This is due to the formation of darker color complex on the silver nitrate impregnated filter paper when

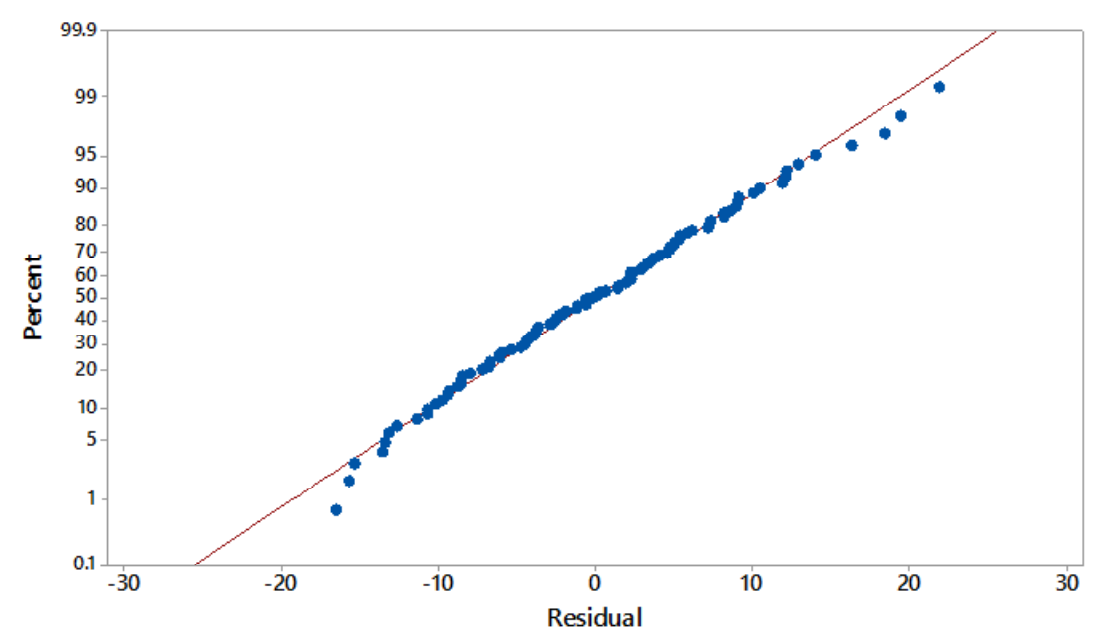

Fig. 8: Normal probability plot of residual values for green color value

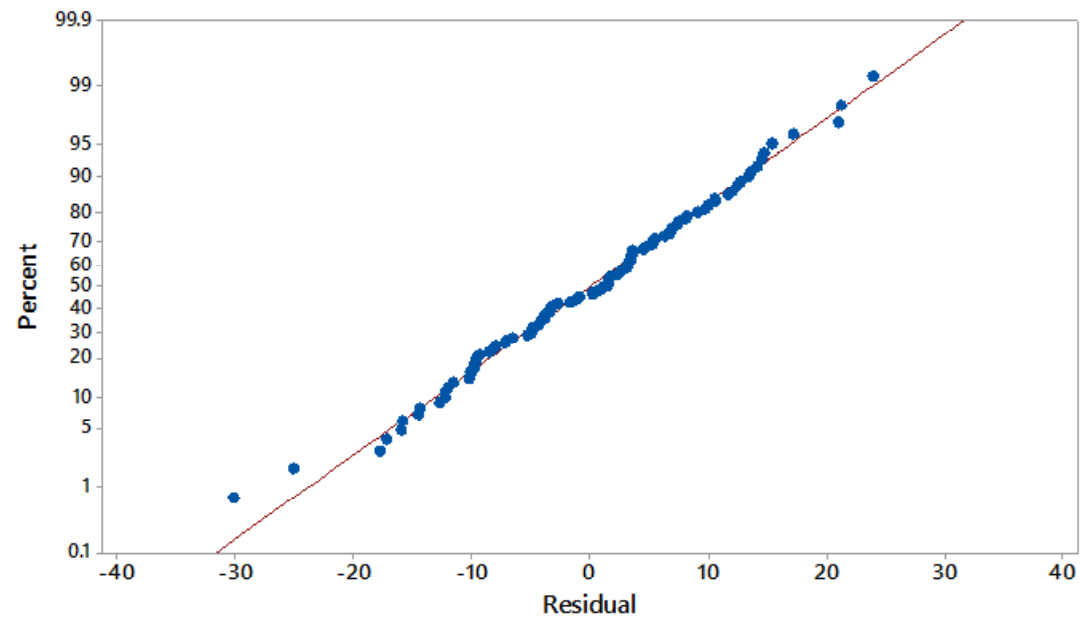

Fig. 9: Normal probability plot of residual values for blue color value 
higher mass ratio of sulfamic acid to zinc powder was applied which resulted in more production of arsine gas which reacts with silver nitrate on the impregnated filter paper. Thus, it can be said that the effect of mass ratio of sulfamic acid to zinc powder is negative in color values, but it is positive effect in detection of arsenic (III) as darker color has lower color value.

Interaction effect of drying weight-drying period of silver nitrate-impregnated filter- paperreaction period (DW $\times$ DP $\times$ RP) interaction and drying weight-mass ratio of sulfamic acid to zinc powder -reaction period (DW x MSZ x RP) interaction
Apart from main effect, interaction effects between the parameters were also investigated in this study and results are presented in Figures 7 to 9. Among all the interaction effects, there were only two of the three interaction effects i.e. drying weightdrying period of silver nitrate-impregnated filterpaper-reaction period (DW x DP x RP) interaction and drying weight-mass ratio of sulfamic acid to zinc powder-reaction period (DW $x$ MSZ $x$ RP) interaction were significantly affect all color values except blue color value on the production of color compunds on the silver nitrate-impregnated filter paper.

\section{Optimisation of Arsenic (III) Detection}

Experiments with various mass ratio of sulfamic acid to zinc powder (MSZ) i.e. $1.0 \mathrm{~g}$ : $0.5 \mathrm{~g}$;

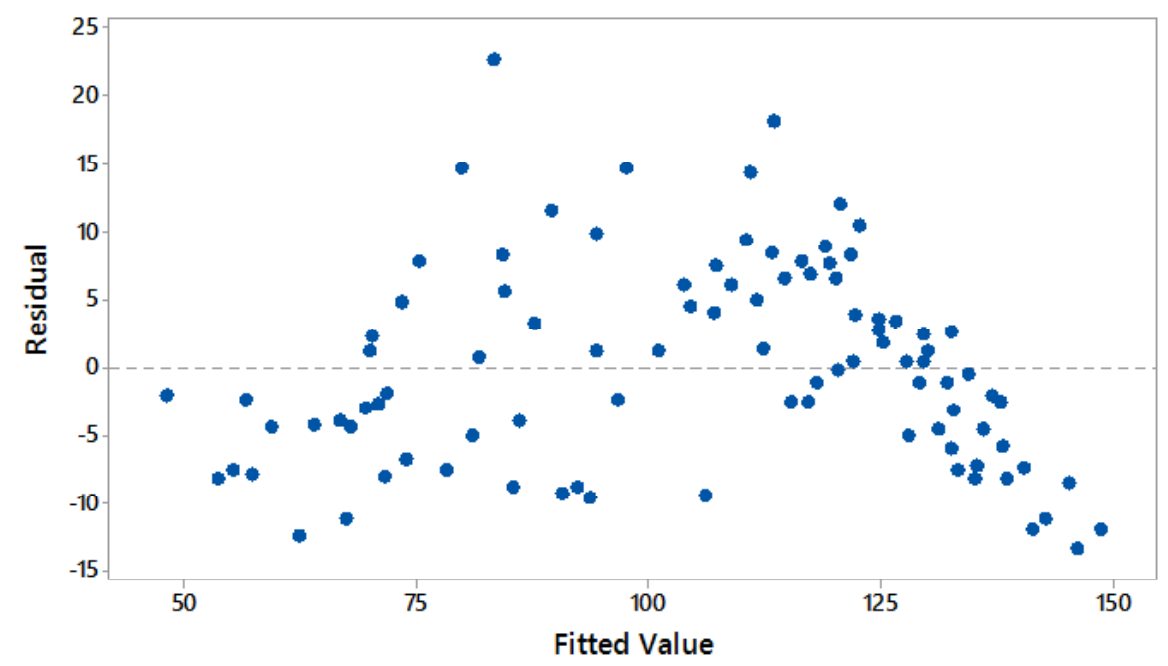

Fig. 10: Residual versus fitted value plot for red color value

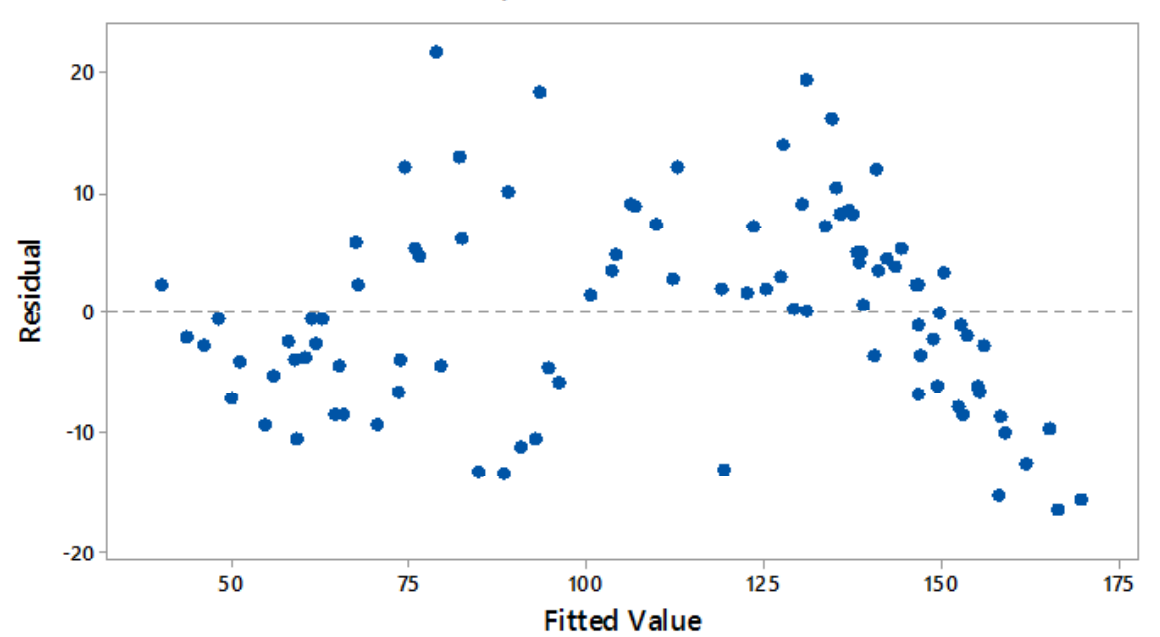

Fig. 11: Residual versus fitted value plot for green color value 
$2.5 \mathrm{~g}: 1.25 \mathrm{~g}$ and $4.0 \mathrm{~g}: 2.0 \mathrm{~g}$, and different reaction periods (RP) ( 5 minutes, 7.5 minutes and 10 minutes) were conducted to validate the optimum conditions by optimisation plot using Minitab software version 17, whereas the weight load used for drying silver nitrateimpregnated filter paper (DW) and drying period of silver nitrate-impregnated filter paper (DP) were fixed at low levels i.e. $100 \mathrm{~g}$ and 20 seconds, respectively, as both were found to be insignificant factors. The optimization plot (Fig. 13) shows the effect of each factor on the responses or composite desirability. The vertical red lines on the graph represent the current factor settings. The numbers displayed in bracket show the current factor level settings (in red). Both horizontal blue dash lines and numbers indicated by $y$ which represents the responses for the current factor level. The plot displays the optimum mass ratio of sulfamic acid to zinc powder (MSZ) and the optimum

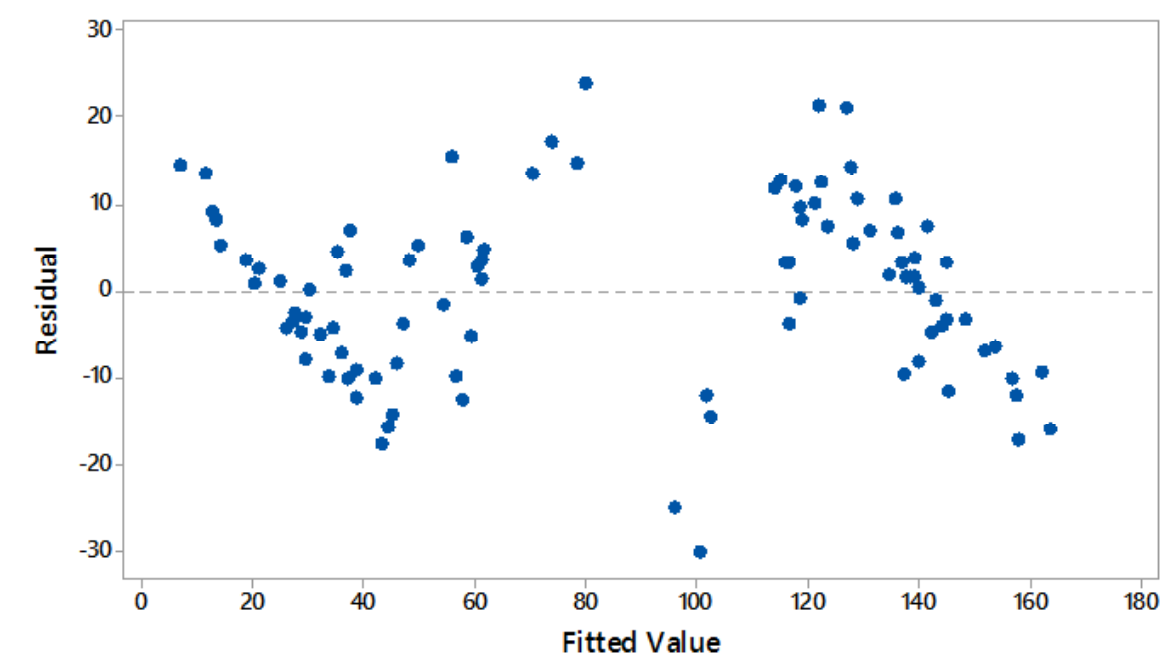

Fig. 12: Residual versus fitted value plot for red color value

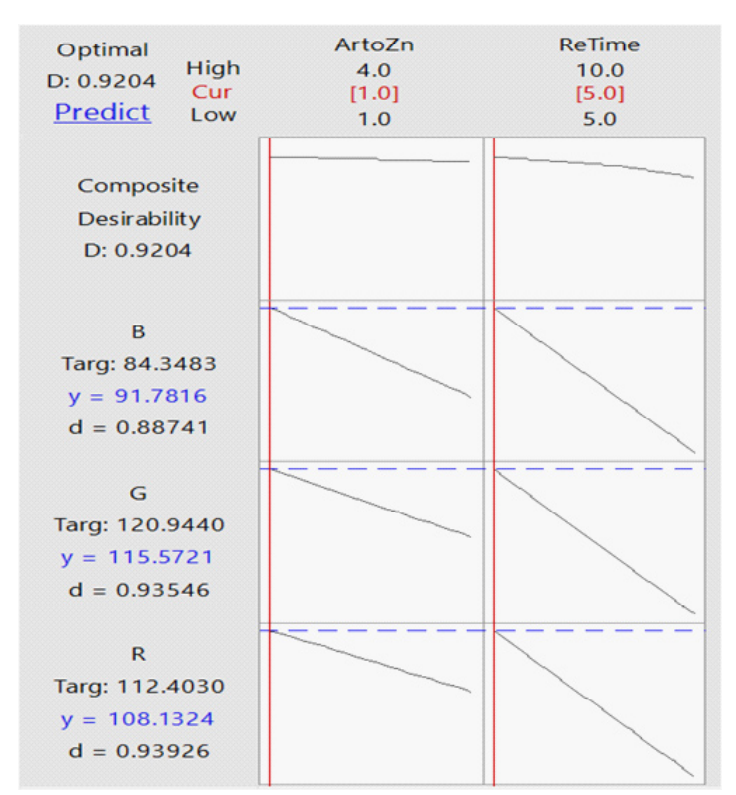

Fig. 13: Optimization plot for Arsenic (III) detection reaction period (RP) were $1 \mathrm{~g}$ of sulfamic acid and $0.5 \mathrm{~g}$ of zinc powder, and 5 minutes, respectively.

\section{Comparison of arsenic detection performance}

Arsenic detection performance of this present method was compared with the methods developed by previous researcher. The performance was evaluated in terms of linear detection range and reaction period. In general, a longer reaction period is required for detection of arsenic using the method developed by previous researcher except the reaction period reported by Shrivas et al. (2015) ${ }^{12}$, which was similar to reaction period found in this study. Previous researcher such as Siangproh et al.(2016) ${ }^{13}$ reported that 7 minutes is required to detect 0.5 to $30 \mathrm{mg} / \mathrm{L}$ of total inorganic arsenic, whereas Huang et al. (2015) ${ }^{14}$ have developed a bacterial biosensor to detect 10 to $500 \mu \mathrm{g} / \mathrm{L}$ of As in 3-h reaction time. Kiso et al. (2015) ${ }^{15}$ documented that a linear range of $0.01-0.1 \mathrm{mg} \mathrm{As} / \mathrm{L}$ for detection of arsenic $(\mathrm{As}(\mathrm{III})$ and $\mathrm{As}(\mathrm{V}))$ in 30 minutes using 
detection tube method. Besides that, a novel wholecell arsenite biosensor was developed using the photosynthetic bacterium Rhodopseudomonas palustris no. 7 for detection of arsenite $(0-500 \mu \mathrm{g} / \mathrm{L})$ after 24 hours (Yoshida et al., 2008) ${ }^{16}$. Das et al. $(2014)^{17}$ used two different kits for detection of 10 $\mu \mathrm{g} / \mathrm{L}-250 \mu \mathrm{g} / \mathrm{L}$ of total arsenic in water in 7 minutes. In addition, the present method was simple, rapid and sensitive compared to the method developed by previous workers.

\section{CONCLUSION}

In this work, various effects were investigated using $2^{4}$ full factorial design for detection of arsenic (III) by colorimetric incorporated with image processing technique. The mass ratio of sulfamic acid to zinc powder was the most significant factor affected RGB color values and followed by reaction period. Drying weight-drying period of silver nitrateimpregnated filter-paper-reaction period (DW x DP $x \mathrm{RP}$ ) interaction as well as drying weight-mass ratio of sulfamic acid to zinc powder-reaction period (DW x MSZ x RP) interaction significantly affected red and green color values, thus significantly influenced the detection. The optimum conditions for detection of arsenic (III) were found to be using $1 \mathrm{~g}$ of sulfamic acid and $0.5 \mathrm{~g}$ of zinc powder at 5 minutes. The present work also demonstrates that the developed method can be used to detect arsenic (III) rapidly and easily.

\section{ACKNOWLEDGMENTS}

The authors thank to Ministry of Higher Education for financing this work via research grant (PRGS/1/2012/STWN01/UPNM/02/1) and Universiti Pertahanan Nasional Malaysia for providing the research facilities.

\section{REFERENCES}

1. Naujokas, M. F.; Anderson, B.; Ahsan, H.; Vasken Aposhian, H.; Graziano, J.H.; Thompson, C.; Suk,W.A.; K. Environ. Health Perspect, 2013, 121(3), 295-302.

2. Nordstrom, D.K. Science, 2002, 296, 2143.

3. Cullen W. R.; Reimer K. J. Chem. Rev. 1989, 89(4), 713-64.

4. Mizumura, A.; Watanabe, T.; Kobayashi, Y.; Hirano, S. Toxicol. Appl. Pharmacol. 2010, 242(2), 119-125.

5. Shen, S.; Li, X.-F.; Cullen, W.R.; Weinfeld, M.; Le, X.C. Chem. Rev. 2013, 113(10), 77697792.

6. Bednar, A.J.; Garbarino, J.R.; Burkhardt, M.R.; Ranville J.F.; Wildeman T.R. Water Res., 2004, 38, 355-364.

7. Wang, Y.; Pan, G.; Wang, K.Q. Nanotech 2011: Technical Proceedings of the 2011 NSTI Nanotechnology Conference and Expo, Boston, MA, 2011, 3, 521-524.

8. Safarzadeh-Amiri, A.; Fowlie, P.; Kazi, A.I.; Siraj, S.; Ahmed, S.; Akbor, A. Sci. Total Environ. 2011, 409, 2662-2667.

9. Anderson, R.D.; McNeill, L.S.; Edwards, M.;
Morton, S.C. J. Environ. Eng. 2008, 134(5), 382-388.

10. Cherukuri, J.; Anjaneyulu, Y. Int. J. Environ. Res. Public Health. 2005, 2(2), 322 - 327.

11. Ong, K. K.; Zainuddin, M.; Teoh, C. C.; Yusof, N. A.; Wan Yunus, W. M. Z.; Mohd Azmi, A. F. Environ. Eng. Manag. J. 2015, 14(8), 19631968.

12. Shrivas, K.; Shankar, R.; Dewangan, K. Sensor. Actuat. B - Chem. 2015, 220, 13761383.

13. Siangproh, W.; Chailapakul, O.; Songsrirote, K. Talanta. 2016, 153, 197-202.

14. Huang, C.-W.; Wei, C.-C.; Vivian Liao, H.-C. Chemosphere. 2015, 141, 44-49.

15. Kiso, Y.; Asaoka, S.; Kamimoto, Y.; Tanimoto, S.; Yokota, K. J. Environ. Chem. Eng. 2015, 3, 40-45.

16. Yoshida, K.; Inoue, K.; Takahashi, Y.; Ueda, S.; Isoda, K.; Yagi, K.; Maeda, I. Appl. Environ. Microbiol. 2008, 74(21), 6730-6738.

17. Das, J.; Sarkar, P.; Panda, J. Pal, P. J. Environ. Sci. Health. A Tox. Hazard. Subst. Environ. Eng. 2014, 49, 108-115. 